\title{
Coronary arterial anatomy in tetralogy of Fallot: morphological and clinical correlations
}

\author{
J Li, N D Soukias, J S Carvalho, S Yen Ho
}

\begin{abstract}
Objective-To clarify the problems in angiographic diagnosis of major coronary arteries crossing the right ventricular outflow tract.

Design-A retrospective study with clinicomorphological correlations to ascertain any aberrant coronary arteries and variations in distribution of the normal right coronary arterial branches.

Setting-Tertiary referral centre.

Subjects-36 necropsy specimens together with the aortograms and surgical reports from 130 patients with tetralogy of Fallot. Results-A preventricular branch was found in $19 \%$ of cases with tetralogy of Fallot, but in none of 13 normal hearts. Aberrant origin of the anterior interventricular coronary artery was found in $14 \%$ of the specimens. The combination of "laid back" and straight lateral views, when reviewed retrospectively, identified this anomaly correctly in nine of 16 patients, with these findings confirmed at surgery in seven patients. A major branch initially thought to cross the outflow tract was shown retrospectively to be an infundibular artery in six, with surgical confirmation in four. It was a preventricular branch in another patient. Conclusions-Using the laid back view alone, infundibular and preventricular branches may be mistaken for a major aberrant artery. A combination of laid back and straight lateral views is needed to avoid false positive diagnosis.

(Heart 1998;80:174-183)
\end{abstract}

1 April 1998

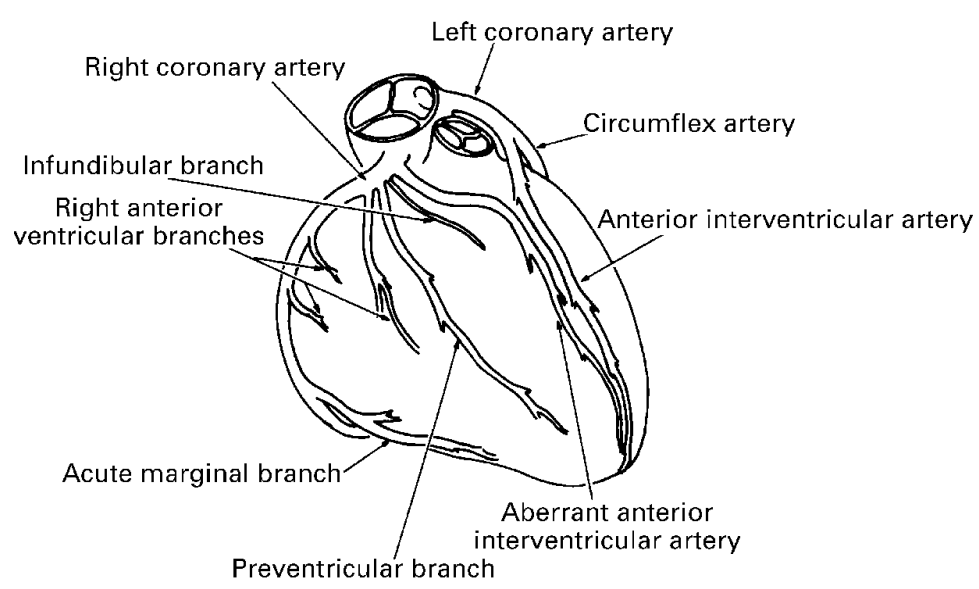

Figure 1 Relations of the various ventricular branches of the coronary arteries as seen on the sterno-costal surface of the heart. The course of the infundibular branch across the subpulmonary outflow tract distinguishes it from the right anterior ventricular branches. The preventricular branch is a prominent branch that passes diagonally across the anterior wall towards the apex. The aberrant anterior interventricular artery passes directly across the subpulmonary outflow tract to reach the interventricular groove and then runs toward the apex.
Keywords: angiography; congenital heart defects; tetralogy of Fallot; paediatric cardiology

The incidence of a major coronary artery crossing the right ventricular outflow tract in tetralogy of Fallot is between $5 \%$ and $12 \% .^{1-7}$ Such an anomalous vessel is not always detectable intraoperatively. ${ }^{3}$ Preoperative recognition of such arteries is therefore crucial in deciding the time and type of operative procedure to be performed. Despite advances in echocardiographic assessment, investigation by angiography is still considered important in evaluating the coronary arterial patterns preoperatively. The sensitivity and specificity of such angiography, however, remains uncertain. Aortography in standard and oblique projections often fails to identify the origins of the coronary arteries and, more importantly, fails to reveal their relation to the right ventricular outflow tract. More recently, the caudo-cranial left anterior oblique (so called "laid back") aortograms have been advocated for identifying abnormal vessels that cross the pulmonary outflow tract. ${ }^{8}{ }^{9}$ In reviewing the experience at our hospital over five years, we have found evidence of one false negative and several false positive diagnoses using the laid back view. These patients with false positive angiographic diagnoses were found, at surgery, to have prominent infundibular or preventricular branches of the right coronary artery rather than anomalous origin of the anterior interventricular (anterior descending) branch of the left coronary artery. These difficulties prompted us to reinvestigate the anatomy of the coronary arteries in tetralogy of Fallot, comparing our findings with the variations in distribution of the branches of the right coronary artery.

\section{Methods}

We examined the distribution of the major coronary arteries in 36 formalin fixed specimens with tetralogy of Fallot from our museum. All the specimens were examined externally before removing the perivascular fatty tissue, where necessary, to expose the epicardial course of the coronary arteries.

In each specimen, we recorded the number, position, and shape of the coronary arterial orifices. The sagittal position of each orifice relative to its respective aortic sinus was measured as the distance to the sinutubular junction.

Besides establishing any anomalous course, we also focused attention on the distribution of the branches of the right coronary artery (fig 1). The diameter of the infundibular branch supplying the musculature of the 


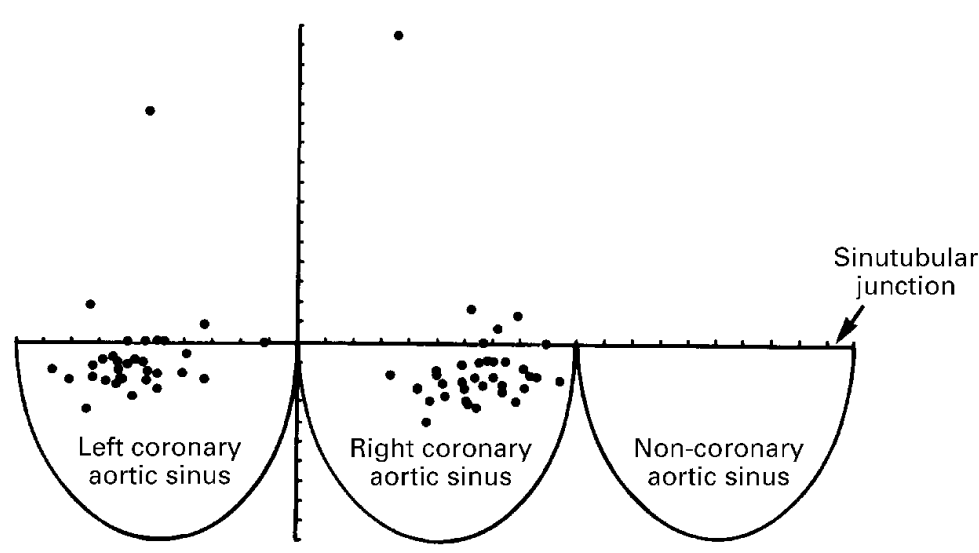

Figure 2 The location of the coronary orifices is plotted in relation to the depth of the sinuses and the distance from the zones of apposition between the facing leaflets. The horizontal and vertical markers represent $10 \%$ of the distances.

Table 1 Data on infundibular branch

\begin{tabular}{llllllll}
\hline & \multicolumn{2}{l}{ Fallot $(n=33)$} & & \multicolumn{2}{l}{ Normal $(n=13)$} & \\
\cline { 2 - 3 } & Range & Mean $(S D)$ & & Range & Mean $(S D)$ & p value \\
\hline Diameter $(\mathrm{mm})$ & 0.8 to 4.0 & $1.66(0.71)$ & & 0.8 to 2.0 & $1.42(0.49)$ & 0.08 \\
Ratio $^{\star}$ & 0.4 to 1.0 & $0.65(0.18)$ & & 0.2 to 0.8 & $0.57(0.17)$ & 0.10
\end{tabular}

^Diameter of infundibular branch:right coronary artery.

subpulmonary infundibulum (excluding the infundibular branch to the ventriculoinfundibular fold) was measured at its origin, and represented as the ratio of its diameter to that of the origin of the right coronary artery. The diameter of the largest right anterior ven-
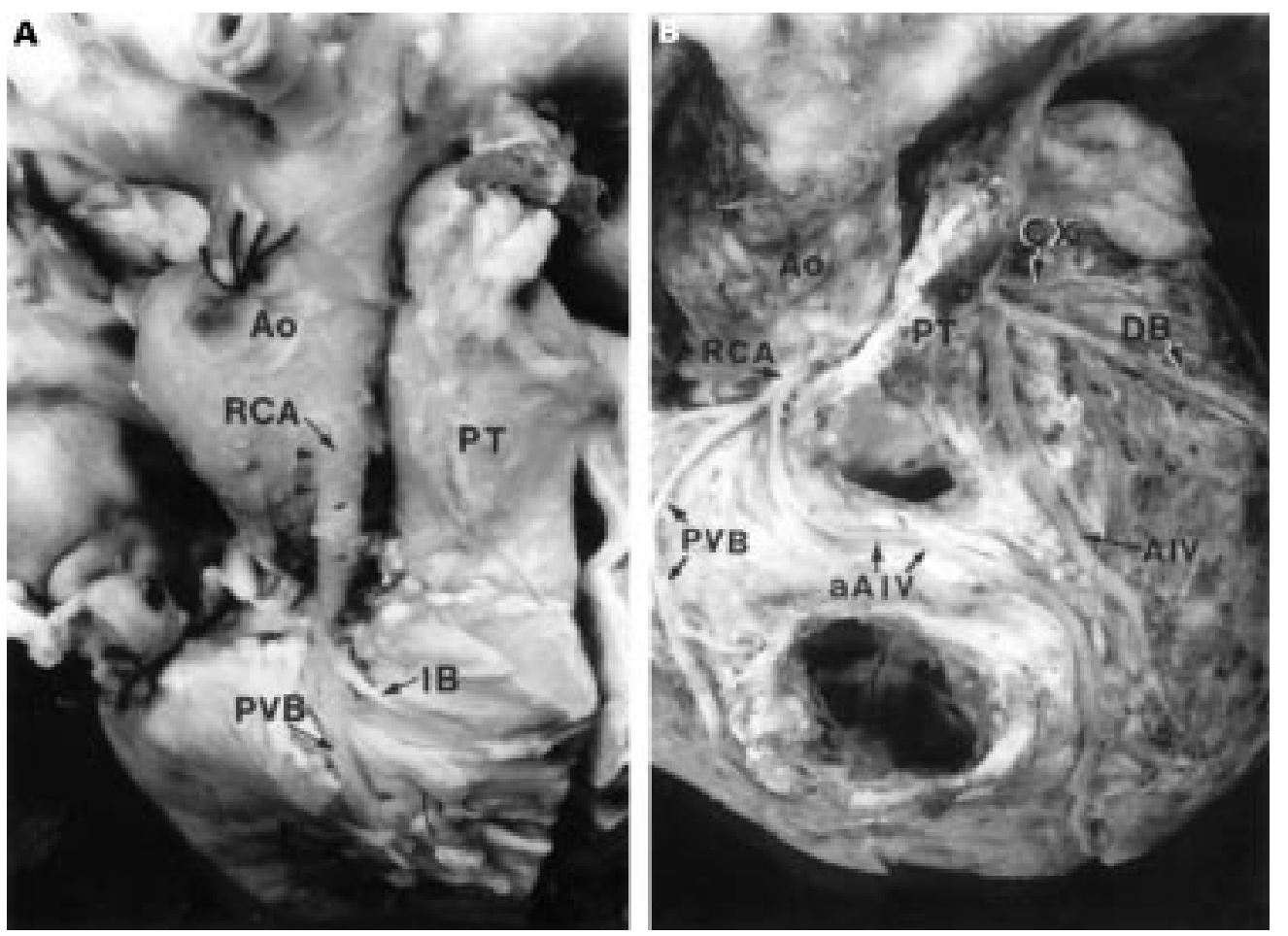

Figure 3 (A) The right coronary artery in this specimen arises well above the sinutubular junction. Its proximal course (between arrowheads) is through the aortic wall. The infundibular branch passes nearly horizontally across the distal right ventricular outflow tract, whereas the preventricular branch passes diagonally across the anterior wall. (B) An aberrant anterior interventricular artery arises from the right coronary artery in this specimen, which had had previous surgical repair. There is an accessory anterior interventricular artery from the left coronary artery, but this accessory artery terminates short of the apex. The preventricular branch is prominent. Ao, aorta; AIV, anterior interventricular artery; a AIV, aberrant anterior interventricular artery; $C x$, circumflex coronary artery; $D B$, diagonal branch; IB, infundibular branch; $P T$, pulmonary trunk; $P V B$, preventricular branch; $R C A$, right coronary artery.

tricular branch was measured at its origin, and again compared with diameter of the right coronary artery itself.

For comparison, we studied 13 normal hearts with a similar size distribution. In these hearts, the length of the infundibular branch was measured from its origin to the termination on the epicardial surface. Data are expressed as mean (SD). Statistical comparisons were made using Student's $t$ test.

Having ascertained the anatomical features in the postmortem hearts, we reviewed the angiocardiograms, which were interpreted pletely blinded to surgical reports, in 130 patients with tetralogy of Fallot undergoing investigation at the Royal Brompton Hospital This is the period over which our unit her the $45^{\circ}$ caudo-cranial, $30^{\circ}$ left anterior oblique angulation view (the so called laid back view), aiming to identify any anomalous course of the coronary arteries. In all patients, we also examined the biplane aortograms to study the distribution of the coronary arteries.

\section{Results}

Age at death had ranged from 3 months to 72 years (median 3 years). The size of the hearts, defined by measuring the distance between the base of pulmonary valve and the apex, varied from 26 to $130 \mathrm{~mm}$ (median $62 \mathrm{~mm}$ ). th 

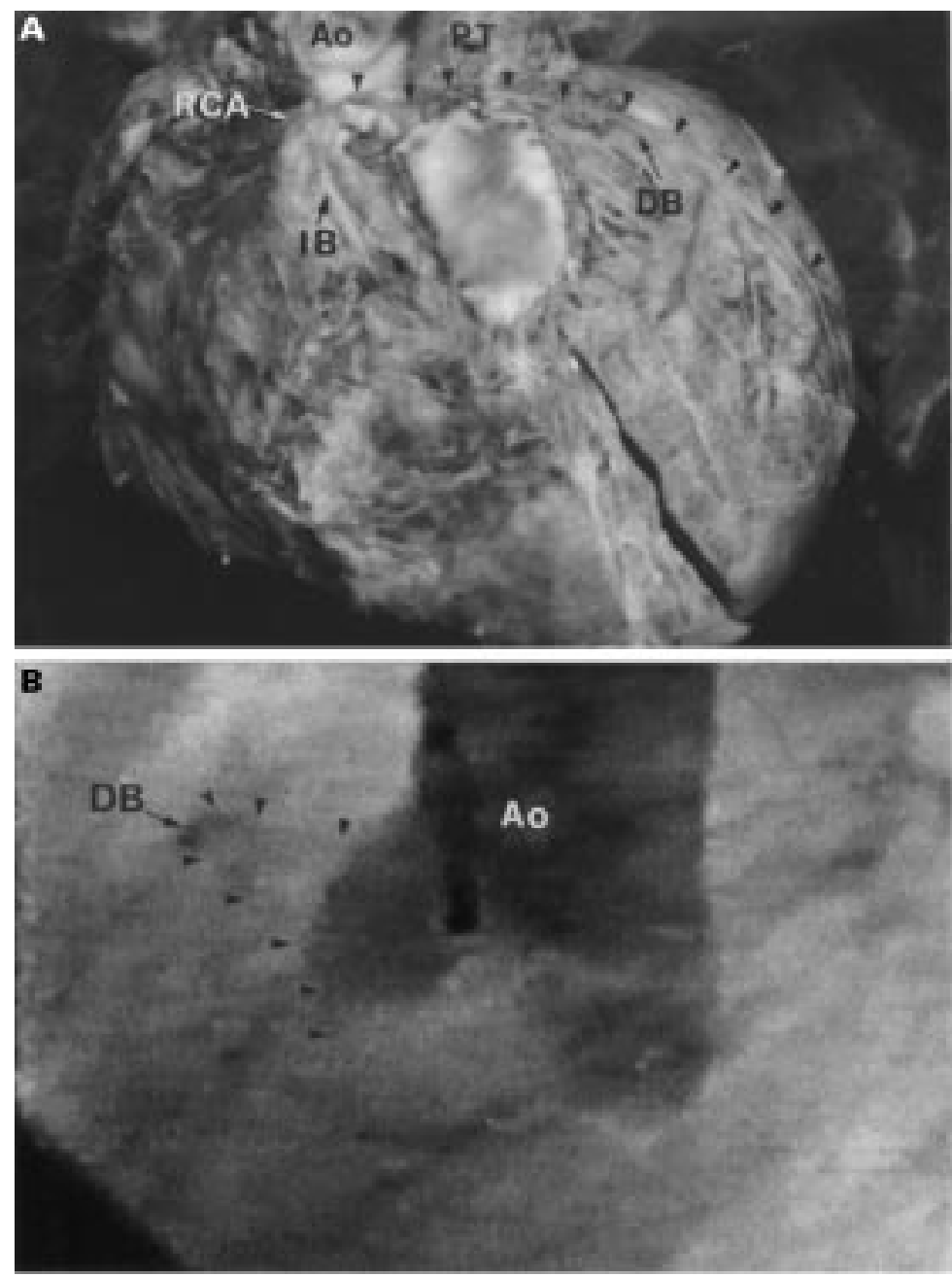

Figure 4 (A) The diagonal artery (arrowheads) passes across the subpulmonary infundibulum. It has been traumatised by sutures securing the outflow patch, resulting in infarction and thinning of the left ventricular wall. The anterior interventricular artery was absent. (B) The aberrant origin of the diagonal branch was not visualised on the atypical laid back view (case 15). Review of the straight lateral view shows the course of the aberrant artery (arrowheads). Ao, aorta; DB, diagonal branch; IB, infundibular branch; PT, pulmonary trunk; $R C A$, right coronary artery.

Table 2 Angiocardiographic and surgical findings of the coronary arteries in tetralogy of Fallot

\begin{tabular}{|c|c|c|c|c|c|}
\hline \multirow[b]{2}{*}{ Case } & \multicolumn{3}{|c|}{ Angiocardiography } & \multicolumn{2}{|l|}{ Surgery } \\
\hline & $\begin{array}{l}\text { Age } \\
\text { (months) }\end{array}$ & Diagnosis (preop) & Diagnosis (rev) & $\begin{array}{l}\text { Age } \\
\text { (months) }\end{array}$ & Diagnosis \\
\hline 1 & 8 & aAIV $<$ RCA & aAIV $<$ RCA & 14 & aAIV $<$ RCA \\
\hline 2 & 5 & aAIV < RCA & IB & & \\
\hline 3 & 9 & aAIV $<$ RCA & $\begin{array}{l}\text { aAIV < RCA, } \\
\text { PVB }\end{array}$ & 12 & $\begin{array}{l}\text { aAIV < RCA, } \\
\text { PVB }\end{array}$ \\
\hline 4 & 6 & aAIV < RCA & aAIV < RCA & & \\
\hline 5 & 15 & $\begin{array}{l}\text { aAIV }<\text { single } \\
\text { RCA }\end{array}$ & $\begin{array}{l}\text { aAIV }<\text { single } \\
\text { RCA }\end{array}$ & & \\
\hline 6 & 36 & aAIV < RCA & aAIV < RCA & $1^{\star}$ & aAIV $<$ RCA \\
\hline 7 & 20 & aAIV < RCA & PVB & & \\
\hline 8 & 4 & aAIV $<$ RCA & IB & 30 & IB \\
\hline 9 & 35 & aAIV $<$ RCA & IB & 60 & IB \\
\hline 10 & 5 & aAIV $<$ RCA & IB & 6 & IB \\
\hline 11 & 3 & aAIV $<$ RCA & aAIV $<$ RCA & 53 & aAIV $<$ RCA \\
\hline 12 & 5 & aAIV $<$ RCA & IB & & \\
\hline 13 & 12 & aAIV $<$ RCA & IB & 43 & IB \\
\hline 14 & 7 & aAIV $<$ RCA & aAIV < RCA & 51 & aAIV $<$ RCA \\
\hline 15 & 83 & Normal & $\mathrm{DB}<\mathrm{RCA}$ & 84 & $\mathrm{DB}<\mathrm{RCA}$ \\
\hline 16 & 6 & Normal & aAIV $<$ RCA, IB & 10 & aAIV $<$ RCA, IB \\
\hline & $36+$ & aAIV $<$ RCA & & & \\
\hline
\end{tabular}

${ }^{\star}$ Age at Blalock-Taussig shunt operation; †age at postoperative angiography.

aAIV, anterior interventricular coronary artery; DB, diagonal branch; IB, infundibular branch preop, before operation; PVB, preventricular branch; RCA, right coronary artery; rev, review; $<$, arising from.
Location of coronary arterial orifices

The origins of the left coronary artery in 32 and the right coronary artery in 34 specimens are shown in fig 2 .

In one specimen in which the orifices of the coronary arteries were $115 \%$ and $154 \%$ of the depth of the sinuses above the sinutubular junction, both arteries ran an intramural course for a distance of $3 \mathrm{~mm}$ (fig 3A). In another three specimens with the orifices just above the junction, the arteries took a very short $(1.5 \mathrm{~mm})$ intramural course at their origin.

\section{Accessory orifices}

Accessory arterial orifices were found in seven specimens, being within the right coronary aortic sinus in six and the left sinus in one. The accessory orifice gave origin to the infundibular branch in two specimens, and in another to a branch dividing into the infundibular branch and a diagonal branch, the latter artery then crossing the outflow tract. In two specimens, two accessory orifices gave origin to infundibular branches. Three accessory orifices were found in one specimen, two of them supplying right ventricular branches and the other an infundibular branch. Separate origins of the circumflex and anterior descending coronary arteries were found in the final specimen.

\section{Obstruction of the orifices}

The orifice of the left coronary artery was potentially obstructed in three specimens: there was a slit in one, the orifice was smaller than the coronary artery in one, and there was atresia of the proximal portion of the left coronary artery in the third. No gross evidence of myocardial infarction was seen in these three specimens.

\section{Distribution of coronary circulation}

A dominant right pattern was present in 24 specimens $(67 \%)$, a dominant left pattern in 10 $(28 \%)$, and a balanced distribution in two $(15 \%)$.

Distribution of the branches of the right coronary artery

The infundibular branch - In 31 specimens, the infundibular branch arising close to the origin of the right coronary artery was of fair size (table 1), and crossed the outflow tract (fig $3 \mathrm{~A})$. In the remaining five specimens, a major anomalous artery arising from the right coronary artery crossed the subpulmonary infundibulum. A separate infundibular branch arose from the origin of the right coronary artery in two, from an aberrant anterior interventricular coronary artery in another, and was lacking in the other two.

The infundibular branch was an obvious and significant vessel in $12(92 \%)$ of the 13 normal hearts, but tiny in the remaining one. In two hearts, the infundibular branch crossed the infundibulum transversely, running just proximal to the base of the pulmonary valve (table $1)$. The mean (SD) length of the infundibular branch was 26.5 (14.2) $\mathrm{mm}$.

The right anterior ventricular branches-One or two prominent right anterior ventricular 

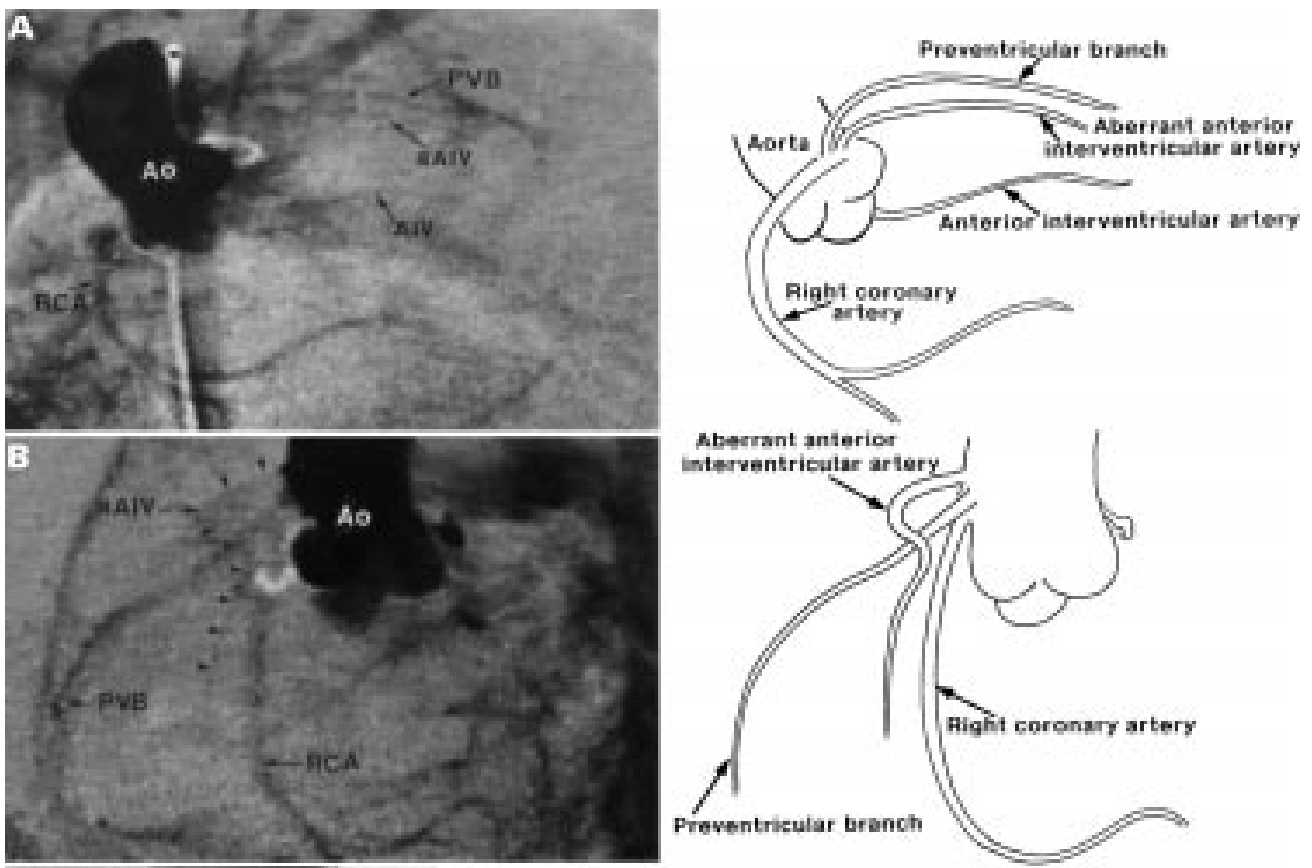

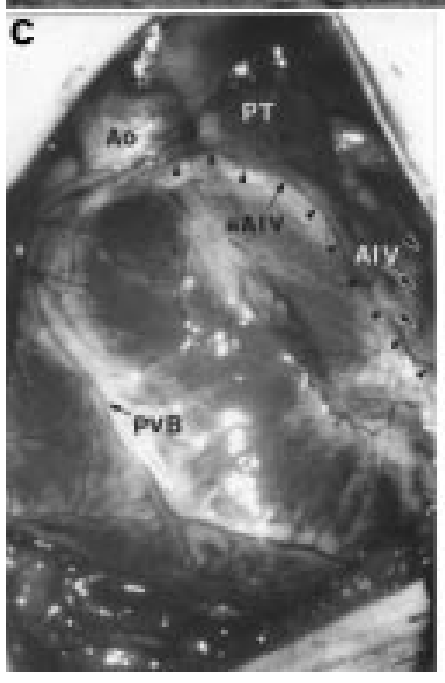

branches arose from the proximal section of the right coronary artery in $15(42 \%)$ of the specimens with tetralogy of Fallot. In two specimens, the right anterior ventricular branch was much bigger than the remaining segment of the right coronary artery, which was tiny. In seven specimens $(19 \%)$, including two with an aberrant anterior interventricular coronary artery crossing the right ventricular outflow tract, the right anterior ventricular branch was extensive, running diagonally along the outflow tract to terminate near the cardiac apex (fig 3B). This branch, which has been named the preventricular branch, ${ }^{10}$ had not been damaged during placement of a patch in the outflow tract in any of these five specimens. In the 13 normal hearts, only one had a large right ventricular branch arising from the proximal portion of the right coronary artery. None had a preventricular branch. Of the specimens with tetralogy of Fallot, $14(39 \%)$ had tiny or no right anterior ventricular branch along the course of the right coronary artery, while three $(23 \%)$ of the normal hearts showed this pattern.
The acute marginal branch - The right coronary artery terminated as the marginal branch in two specimens with tetralogy of Fallot. Otherwise there was no significant difference in the distribution and the size of this artery between specimens of tetralogy of Fallot and normal hearts.

Anomalous coronary arteries in tetralogy of Fallot Unusual pattern - In one specimen, we found two separate right coronary arteries coursing within the right atrioventricular groove (we were unable to establish the nature of their orifices in the aortic sinus). One artery gave rise to the infundibular branch, a right ventricular branch, and a branch to the right atrium. The second artery gave rise to the acute marginal branch and terminated in the posterior interventricular groove.

Atresia of the proximal anterior interventricular coronary artery - This arrangement was found in one specimen. The distal part of the artery was of normal size although collateral supply could not be traced by gross dissection. The 

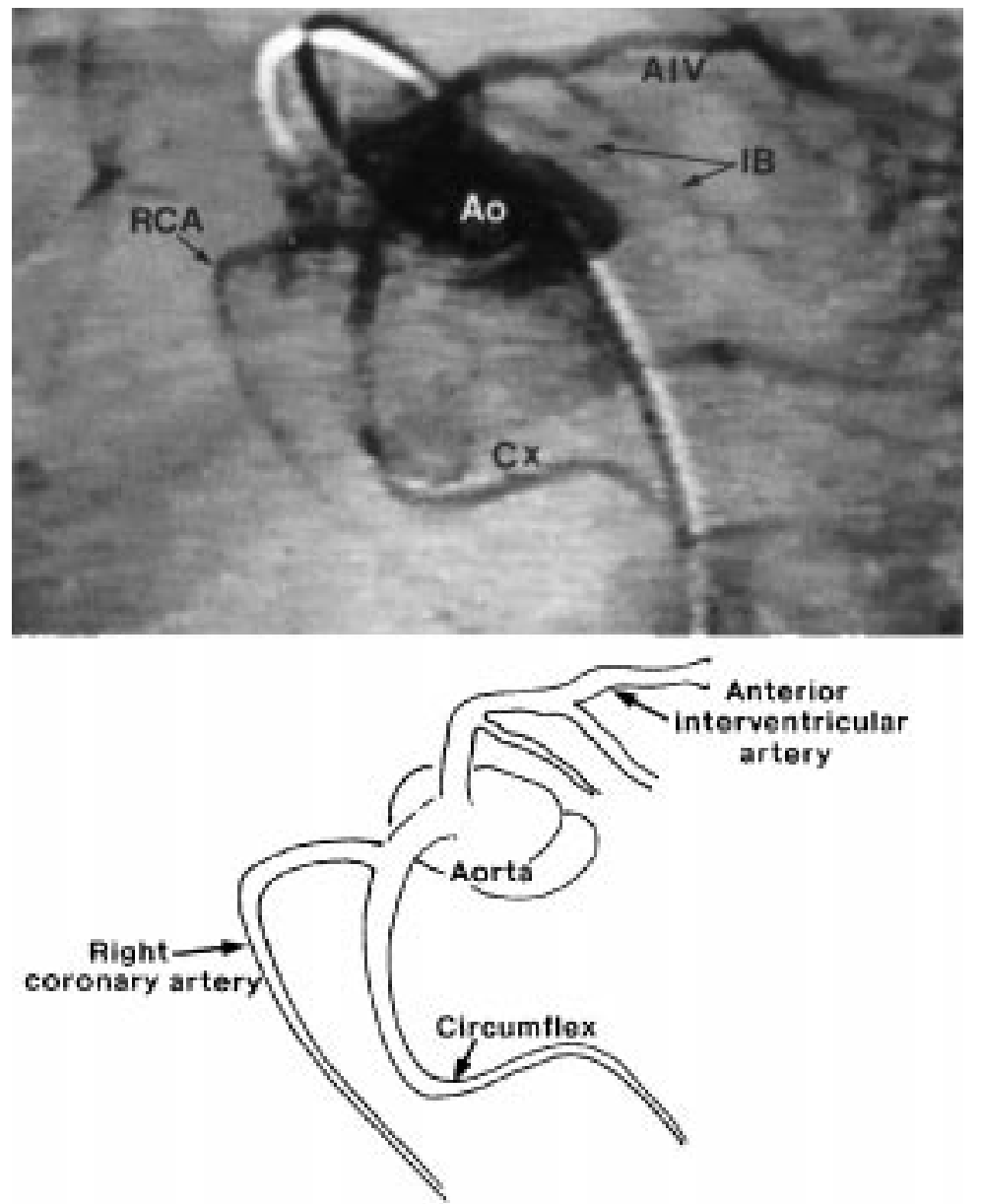

Figure 6 This "laid back" view from case 5 shows the branches from the single coronary artery. The diagram is composed from observations from a series of consecutive frames. The anterior interventricular artery passes from right to left across the subpulmonary infundibulum. Ao, aorta; AIV, anterior interventricular artery; $C x$, circumflex coronary artery; IB, infundibular branch; RCA, right coronary artery.

right coronary artery in this case was normal. Anomalous distribution relative to the subpulmonary infundibulum - A major branch arose from the right coronary artery and crossed the subpulmonary infundibulum in five $(14 \%)$ of the hearts. In three of these specimens, it was the anterior interventricular coronary artery which arose from the proximal portion of the right coronary artery and crossed the outflow tract to reach the interventricular groove (fig 3B). The distance between the aberrant artery and the base of the pulmonary valve was 10,12 , and $25 \mathrm{~mm}$ respectively, with the largest distance being in a large heart measuring $105 \mathrm{~mm}$ from the base of the pulmonary valve to the apex. An additional anterior descending coronary artery, arising from the left coronary artery and running its normal course, was found in these three specimens. This artery was tiny in one, and of the same size as the artery arising from the right coronary artery in the other two (fig 3B).

A solitary artery arising from the right coronary aortic sinus was found in one specimen. The common stem branched, with one of the stems taking the normal course of the right coronary artery. The second turned to the left, crossed the subpulmonary infundibulum, and branched into the anterior interventricular coronary artery, which ran along the interventricular groove, and the circumflex artery, which coursed within the left atrioventricular groove. The distance between the base of the pulmonary valve and main stem of the left coronary artery was $7 \mathrm{~mm}$ in this specimen, which measured $75 \mathrm{~mm}$ to the apex of the heart.

There were two orifices within the right coronary aortic sinus in the remaining specimen. One of these gave rise to the right coronary artery, and the other to an artery which divided into preventricular and diagonal branches. The diagonal branch crossed the subpulmonary infundibulum just beneath the base of the pulmonary valve and extended onto the anterolateral wall of the left ventricle (fig 4A). An artery arising from the left coronary aortic sinus gave rise to the circumflex artery. A small branch arose from the circumflex artery and supplied the ventricular septum. The diagonal branch had been traumatised during the surgical correction (fig $4 \mathrm{~A}$ ).

\section{CLINICAL FINDINGS}

We reviewed the angiocardiograms and surgical records of all 130 patients (69 male and 61 female). Age at the time of angiography varied from 6 days to 8 years (median 11 months). Of these, the laid back view had not been obtained satisfactorily in 16 patients, either because standard angulation had not been appropriate, or because injection into the coronary arteries had been unsatisfactory. In all 130 patients, 116 had been diagnosed preoperatively as having normal coronary arteries. In two of the 116 patients, however (table 2, cases 15, 16; aged 10 and 83 months), an aberrant major coronary artery arising from the right coronary artery and crossing the outflow tract was discovered by the surgeon at operation. One of these patients (case 16) had been studied preoperatively only with aortograms performed using standard views, but had then undergone laid back angiography postoperatively. The second child (case 15) died of poor left ventricular function postoperatively following infarction caused by transection of an undiagnosed aberrant diagonal branch (fig 4).

The diagnosis of an aberrant artery arising from the right coronary artery and crossing the subpulmonary outflow tract had been made following preoperative review of the angiocardiograms in 14 patients (table 2). The decision was made to delay the surgical correction in 11 of these patients (cases 2, 4-9, 11-14), constructing instead a Blalock-Taussig shunt to provide palliation, with or without balloon dilatation of the outflow tract. Eight patients (cases 1, 3, 8-11, 13, 14) have since undergone total surgical correction at ages ranging from six to 60 months.

When reviewing the aortograms retrospectively, we first analysed the laid back view. In the 14 patients (cases $1-14$ ) in whom the preoperative studies suggested an aberrant anterior interventricular coronary artery, the laid back view had been obtained satisfactorily. From this view, we recognised an artery arising from the right coronary artery and crossing the area presumed to be the subpulmonary infundibulum 

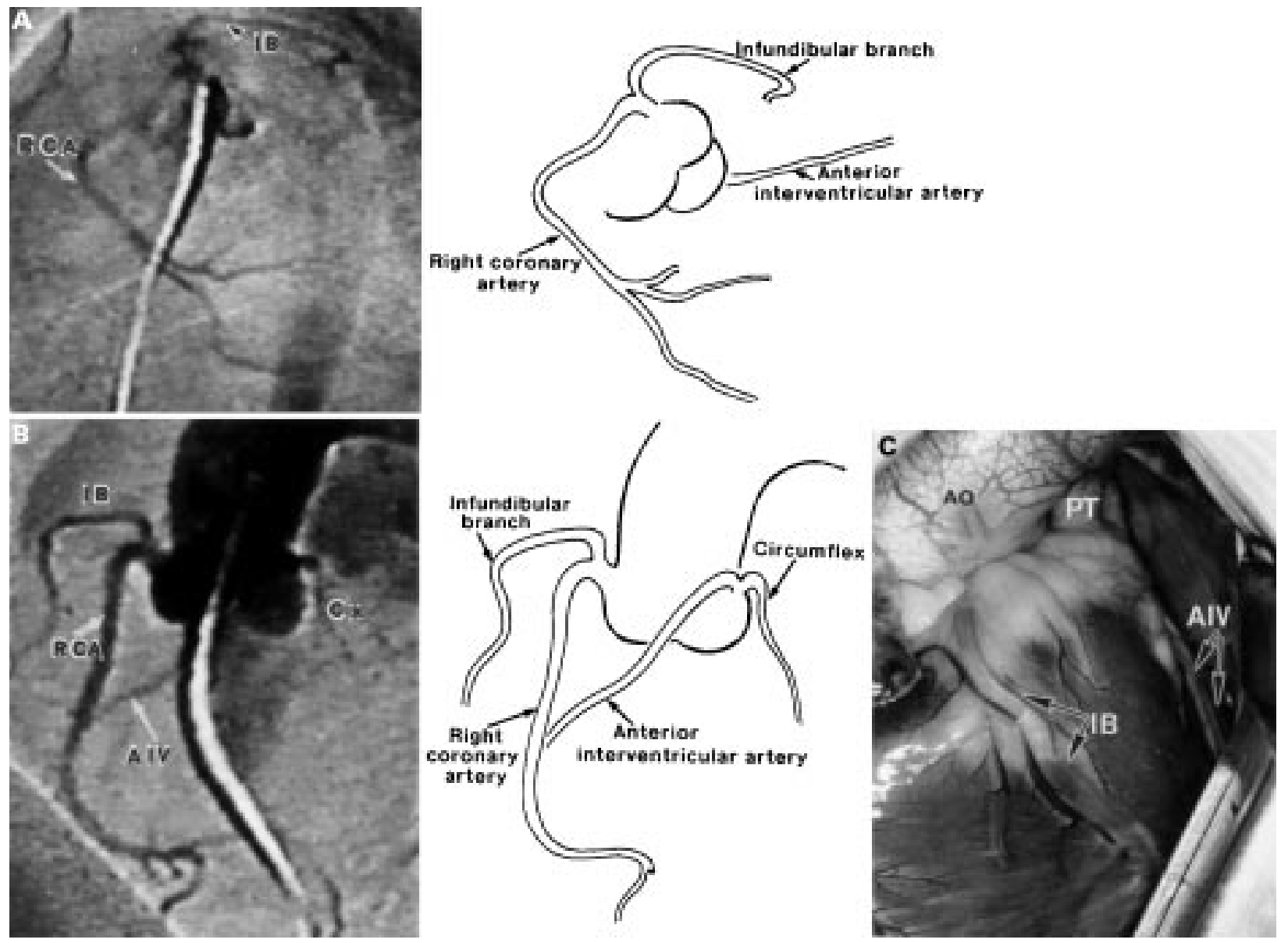

Figure 7 (A) The "laid back" view from case 8 gives the impression of an artery crossing antero-superiorly over the right ventricular outflow tract. (B) The straight lateral view clearly shows this to be the infundibular branch, distant from the normal anterior interventricular artery. (C) The intraoperative view confirms the findings. The infundibular branch crosses the subpulmonary infundibulum, and does not meet the normal anterior interventricular artery. Ao, aorta; AIV, anterior interventricular artery; IB, infundibular branch; PT, pulmonary trunk; RCA, right coronary artery.

in all. In one of them (case 3), two vessels arose from the right coronary artery and crossed anterosuperiorly, almost following the same course (fig 5A). One vessel was large and had been diagnosed as an aberrant anterior interventricular coronary artery preoperatively. The other was small and had not been identified before the operation. In the patient (case 5) with the single right coronary artery, the laid back view showed a common stem arising from the right aortic sinus and dividing into two arteries. One was the right coronary artery, which followed a normal course. It gave rise to the circumflex artery, which passed behind the aorta and ran into the posterior interventricular groove. The second was the anterior interventricular coronary artery, which turned to the left across the outflow tract and gave rise to an infundibular branch (fig 6). In the two patients in whom an aberrant artery was only revealed at operation, the angulation for the laid back view was inadequate in one (case 15). In this view, the diagonal branch arising from the right coronary artery was overlapped by the aorta. The laid back view performed postoperatively in the second patient (case 16) clearly demonstrated the aberrant artery.

By combining the results from the laid back view with the straight lateral view which was obtained with the same injection, more information was obtained in all these 16 patients. In six patients (cases $2,8-10,12,13$ ), the presumed aberrant artery, having arisen from the proximal portion of the right coronary artery, was seen to run anteriorly and inferiorly, not extending towards the base of the pulmonary valve, and failed to reach the anterior interventricular groove (fig 7). This vessel could then be identified as an infundibular branch, being smaller in diameter than the right coronary artery in all these patients. This interpretation has since been confirmed at surgery in four patients (cases $8-10,13)$. Among these four patients, the surgical correction had been delayed in three (cases $8,9,13$ ) until the age of 30,43 , and 60 months, respectively. In one patient (case 7), the combination of angiographic views revealed that the long vessel which arose from the origin of the right coronary artery extended anteriorly and inferiorly to reach almost to the apex, but did not cross the subpulmonary outflow tract. This course enabled it to be diagnosed as a preventricular branch. In only nine patients, therefore (cases 1, 3-6, 11, 14-16), did our retrospective review using combined views identify an anomalous course of a major artery crossing the subpulmonary outflow tract. The presence of an aberrant artery has now been confirmed at surgery in 


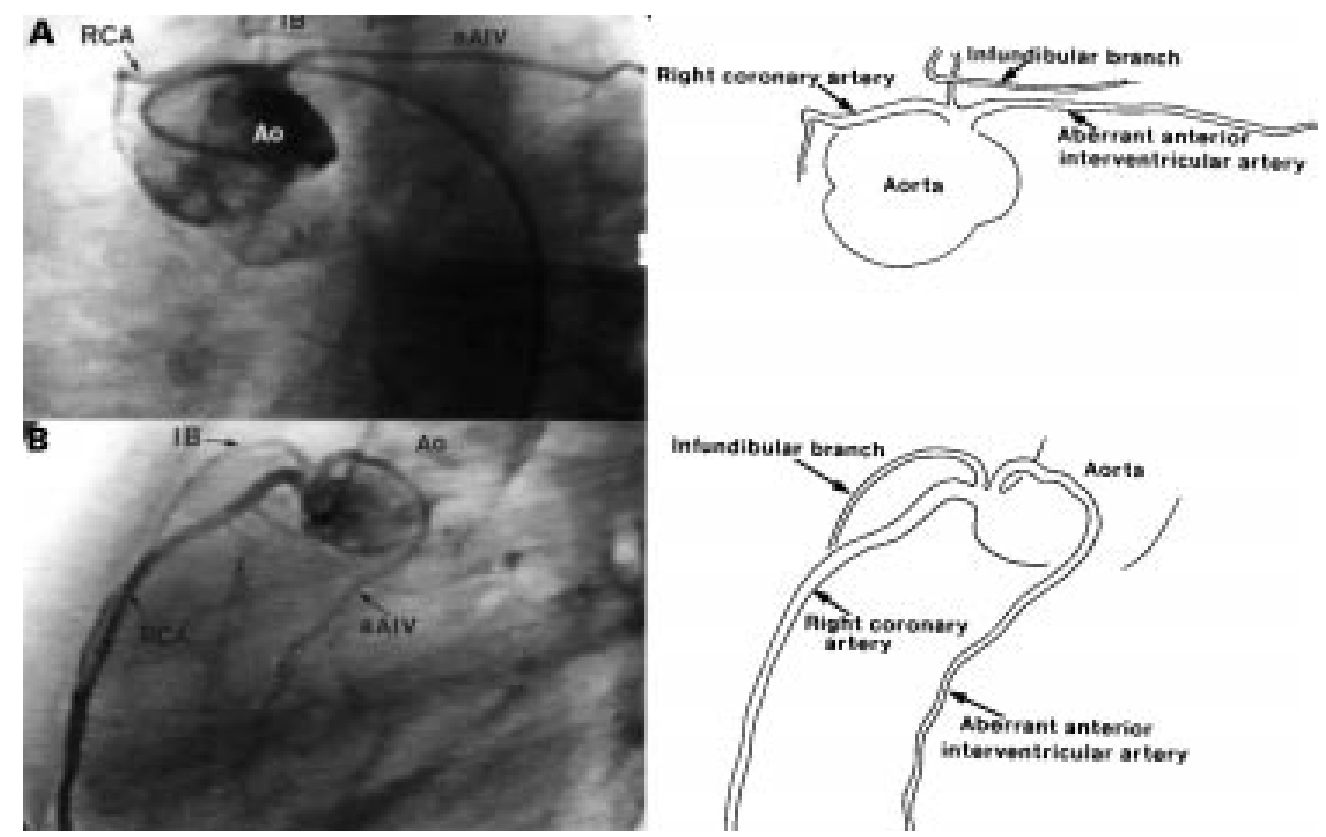

Figure 8 (A) The "laid back" view from case 16 shows two arteries passing antero-superiorly in a similar course. (B) The straight lateral view separates these two arteries into the infundibular branch and the aberrant anterior interventricular artery. The course of the aberrant artery was confirmed at surgery. In this projection, the infundibular branch passes forwards whereas the aberrant artery curves backwards and turns inferiorly. Ao, aorta; aAIV, aberrant anterior interventricular artery; IB, infundibular branch; RCA, right coronary artery.

seven of these nine patients (cases $1,3,6,11$, 14-16), including one (case 6) in whom the pericardium was opened during construction of a left Blalock-Taussig shunt. In the straight lateral view, the anterior interventricular coronary artery, after arising from the origin of the right coronary artery, was shown to turn posteriorly across the outflow tract, and then descend along the anterior interventricular groove (fig 8). In one of the patients (case 3), in whom two vessels were seen crossing anterosuperiorly on the laid back view (fig $5 \mathrm{~A}$ ), the straight lateral view showed that the bigger artery was the preventricular branch while the smaller one was the aberrant anterior interventricular coronary artery (fig 5B). This arrangement was also confirmed subsequently at surgery (fig 5C). An accessory anterior interventricular coronary artery arising from the left coronary aortic sinus and running a normal course was seen in all eight patients with aberrant arteries. Where the diagonal branch was the aberrant artery (case 15), its course as visualised on the lateral view was not significantly different from that of an aberrant anterior interventricular artery (fig 4B).

In 114 patients identified as having normal coronary arteries in retrospective review, the infundibular branch was seen in 96 patients $(84 \%)$, the preventricular branch in seven $(6 \%)$, and a right anterior ventricular branch arising at the origin of the right coronary artery in $17(15 \%)$. This right anterior ventricular branch, when seen in the laid back view, fails to cross the right ventricular outflow tract. In the straight lateral view, it runs a similar course as the preventricular branch-that is, anteriorly and inferiorly.

\section{Discussion}

THE ANATOMY OF CORONARY ARTERIES

The coronary arterial orifices

The location of coronary arterial orifices relative to the aortic sinuses has been extensively studied in the normal hearts. ${ }^{10-12}$ Little attention, however, has been paid to this feature in congenitally malformed hearts, except for the particular condition of common arterial trunk. ${ }^{13}$ When we focused on this feature in tetralogy of Fallot, we found that the orifice of the left coronary artery was located more posteriorly than in normal hearts $^{10}{ }^{11}\left(\mathrm{p}<0.001^{11}\right.$ and $\left.\mathrm{p}<0.05^{10}\right)$. This is probably a result of the abnormal development, ${ }^{12}$ which produces the anterior and rightward position of the aorta. In addition, an intramural course of the origin of a coronary artery - not previously reported to our knowledge in tetralogy of Fallot-was found to be associated with elevation of the orifices above the sinutubular junction in four of our specimens, the greatest severity being seen in one with a very high take off. Such intramural courses have been reported sporadically in normal patients, ${ }^{14-17}$ and are now known to be particularly important in complete transposition because of their implications during the arterial switch operation. ${ }^{18-20}$ Their presence-together with an acute angle and course of a coronary artery - is known also to be associated with sudden death, especially during exercise. ${ }^{14-17}$ High take off may also give difficulties during aortic cross clamping at operation, as well as producing problems during selective arteriography. ${ }^{10}$

The incidence of multiple orifices differed from that in normal series. ${ }^{10-12}$ It is significant that obstruction of the left coronary arterial orifices was found in three specimens (10\%). In one of these the orifice was atretic, as was the 
proximal segment of the anterior interventricular coronary artery. The territory normally supplied by this artery was presumably fed by intramyocardial collaterals. This lesion is also known to be associated with sudden cardiac death.

\section{Distribution of coronary circulation}

The incidence of a dominant left coronary arterial pattern $(28 \%)$ in tetralogy of Fallot is greater than in normal subjects $(10-12 \%) .^{21-23}$ This finding is in keeping with another study, ${ }^{3}$ but has no obvious explanation.

\section{Distribution of the branches of the right coronary artery}

The infundibular (or conus) branch is defined as the vessel that arises either from the origin of the right coronary artery or directly from the right coronary aortic sinus, and supplies the musculature of the subpulmonary infundibulum. Its appearance in tetralogy of Fallot was as seen in most of the normal hearts $(92 \%)$. This finding differs from previous necropsy investigations, which described the artery as "unusually prominent" in all cases, ${ }^{6}$ and "remarkably long" in $40 \%$ of cases. ${ }^{7}$ Another study of normal hearts showed the artery to be similarly significant in about half the cases examined. ${ }^{10}$

The anterior right ventricular branches arise from the right coronary artery along its course and supply the anterior wall of the right ventricle. A well recognised variation was seen in 15 $(42 \%)$ of our specimens, with one or two large branches arising from the origin of the right coronary artery and running diagonally to supply the anterior wall of the right ventricle. In seven specimens with tetralogy (19\%), a prominent branch described as a preventricular branch was recognised. This branch could be confused with an aberrant anterior interventricular artery.

Anomalous distribution of coronary arteries In $14 \%$ of our series, we discovered a major aberrant artery arising from the right coronary artery which crossed the right ventricular outflow tract. All the aberrant arteries ran close to the base of the pulmonary valve. Modifications of incisions into the outflow tract, use of a conduit from the right ventricle to the pulmonary trunk, or more recently the transatrial and transpulmonary approach, have all been advocated as means of avoiding these aberrant arteries. ${ }^{24} 25$

DIAGNOSTIC PROBLEMS WITH THE LAID BACK VIEW Tetralogy of Fallot is one of the conditions for which angiography is still performed before surgical correction in many centres. Although there is increasing experience of echocardiographic assessment of patients with tetralogy of Fallot, ${ }^{26}{ }^{27}$ abnormalities of pulmonary and coronary arteries are not always identified. The sensitivity and specificity of echocardiographic diagnosis of coronary abnormalities in clinical practice is unclear. ${ }^{25}$ Thus angiography still plays an important role in the evaluation of aberrant coronary arteries in tetralogy of Fallot.
The newly advocated laid back projection of the aortogram was first described for use in patients with disease of the aortic valve. ${ }^{29}$ It was then used in complete transposition. ${ }^{30}$ In recent years, it has been used in tetralogy of Fallot, specifically to identify the anomalous coronary arteries which cross the right ventricular outflow tract. ${ }^{8}$ It has been suggested that, because in this projection the outflow tract is anterior and to the left of the aortic valve, any vessel coursing in this area should be considered as crossing the outflow tract. When performed correctly, false negative diagnosis should be avoidable. Several false positive diagnoses, however, have ensued in our experience with this method over the past five years. These spurious diagnoses have been confirmed at surgery thus far in five patients, the artery identified by the surgeon proving to be a prominent infundibular branch in four and a preventricular branch in the other. These findings are no more than expected, since examination of our postmortem specimens shows that the infundibular branch does indeed cross the outflow tract. It does not, however, enter the anterior interventricular groove. When the infundibular branch is long, and only the laid back view is examined, it is difficult to differentiate from an aberrant anterior interventricular coronary artery arising from the right coronary artery and crossing the outflow tract. Furthermore, with the hearts available to us we found that the presumed subpulmonary infundibulum - as seen in the laid back view - also included part of the apical trabecular component of the right ventricle. This is because the ventricular mass is markedly foreshortened in this projection. Because of this, the long preventricular branch can also be mistaken for a major vessel crossing the outflow tract when the laid back view is used in isolation.

Knowing the factors which can result in a false positive diagnosis in the laid back view, we combined retrospective examination of this view with the available straight lateral projections of the angiograms. It was always the straight lateral view that was traditionally recommended for analysis of the coronary arterial anatomy in tetralogy of Fallot. ${ }^{4}$ In this projection, the origins of the right and left coronary arteries are well separated, since the counterclockwise aortic rotation (viewed from above) in tetralogy results in the right and left coronary aortic sinuses having an almost direct anterior-posterior relation. There is also no caudal or cranial angulation in the straight lateral view. Thus the course-and more importantly the relation - between any of these arteries and the outflow tract, together with the base of pulmonary valve, can be directly identified. In this projection, the infundibular branch runs anteriorly and inferiorly, and then turns around the outflow tract, but does not meet the anterior interventricular coronary artery at the interventricular groove. The preventricular branch runs anteriorly and inferiorly and reaches nearly to the apex, but without turning around the outflow tract. The aberrant anterior interventricular coronary artery, in contrast, 
having arisen from the right coronary artery, extends immediately across the outflow tract. It then approaches the base of pulmonary valve before entering the anterior interventricular groove, which tends to be displaced posteriorly because of the considerable clockwise rotation of the interventricular septum in tetralogy of Fallot. Within the interventricular groove, it meets the accessory anterior interventricular coronary artery, if such an artery is present.

When reviewing the aortograms retrospectively in this fashion in 16 patients, only nine were identified as having an anomalous artery. In six of these, our retrospective findings have been confirmed at surgery. Another had a preventricular branch together with an aberrant anterior interventricular coronary artery crossing the outflow tract. This finding was also confirmed at surgery. The remaining two patients are still awaiting their operations, including the patient with the single right coronary artery. In the two patients in whom an aberrant artery had not been identified preoperatively following examination of standard views and an atypical laid back view, retrospective review of the straight lateral projection clearly showed its course across the outflow tract into the anterior interventricular groove. When an aberrant anterior interventricular coronary artery (or any other major vessel) crossing the outflow tract coexists with an infundibular branch or preventricular branch, therefore (as seen in cases 3 and 16, and in five specimens), it is even more important to examine the straight lateral view, since these arteries can seem to follow the same course when seen in the laid back view.

In clinical practice, it is crucial to differentiate normal branches of the right coronary artery from the anterior interventricular coronary artery, or from any other major coronary artery crossing the outflow tract. The infundibular branch was prominent in all our specimens of tetralogy of Fallot. It was also seen in $92 \%$ of our normal hearts, and was recognised in half the normal hearts examined in another study. ${ }^{10}$ Such infundibular branches, however, do not represent a contraindication to ventriculotomy in the correction of tetralogy unless they are equal in calibre, or larger than, the right coronary artery. ${ }^{1-3}$ Such equality was seen in only one of our specimens, and in none of our patients. The preventricular branch does not cross the outflow tract, and so does not influence surgical correction. It is only the aberrant anterior interventricular coronary artery, or any other major artery arising from the right coronary artery which crosses the outflow tract, that presents a serious hazard if unrecognised at the time of right ventriculotomy. Even the recently developed transatrial-transpulmonary approach still requires a patch across the ventriculo-pulmonary junction in about half of the cases. ${ }^{25}{ }^{31}$ When recognised, the surgical options are to modify the approach or to delay the age of correction. In cases of incorrect diagnosis, however, unnecessary delay may ensue, as occurred in three of our patients, but thus far without deleterious consequence.
LIMITATIONS OF THE STUDY

We have seen only a relatively small number of specimens to document the variability in location of the coronary arterial orifices and the distribution of the branches of the right coronary artery. The fixation of these specimens can affect the shape and size of the coronary arterial orifices and branches, but should not influence their arrangement nor the ratio between the branches and their main stems. The infundibular branch had been incorporated in an outflow patch during complete surgical correction in 15 of 36 specimens. It may have been more prominent in these hearts. Furthermore, the retrospective study of the angiograms has been confirmed by surgery thus far in only 11 of 16 patients.

\section{CONCLUSION}

Infundibular and preventricular branches may be mistaken for a major coronary artery arising from the right coronary artery and crossing the right ventricular outflow tract. The laid back view is an excellent means of identifying all these vessels, but cannot consistently distinguish between them. To achieve distinction of the major anomalous arteries, it is necessary to combine the laid back view with the straight lateral view. This study was supported by the British Heart Foundation. We
are indebted to Prof Andrew Redington, Dr Michael Rigby, and Dr Elliot Shinebourne, who looked after these patients and performed the angiographic studies; and to $\mathrm{Mr}$ Christopher Lincoln and Mr Darryl Shore who performed the surgical proLincoln and Mr Darryl Shore who performed the surgical pro-
cedures. In particular, we thank Prof Robert H Anderson for his cedures. In particular, we thank Prof Rob
guidance and review of the manuscript.

1 Dabizzi RP, Teodori G, Barletta GA, et al. Associate coronary and cardiac anomalies in the tetralogy of Fallot. Angiographic study. Eur Heart $\mathcal{F}$ 1990;11:692-704.

2 Shrivastava S, Mohan JC, Mukhopadhyay S, et al. Coronary artery anomalies in tetralogy of Fallot. Cardiovasc Intervent Radiol 1987;10:215-18.

3 Dabizzi RP, Caprioli G, Aiazzi L, et al. Distribution and anomalies of coronary arteries in tetralogy of Fallot. Circu-

4 Fellows KE, Freed MD, Keane JF, et al. Results of routine preoperative coronary angiography in tetralogy of Fallot. Circulation 1975;51:561-6.

5 Hurwitz RA, Smith W, King H, et al. Tetralogy of Fallot with abnormal coronary artery: 1967 to 1977 . $\mathcal{F}$ Thorac Cardiovasc Surg 1980;80:129-34.

6 Longenecker CG, Reemtsma K, Creech O. Anomalous coronary artery distribution associated with tetralogy of Fallot: a hazard in open cardiac repair. $\mathcal{F}$ Thorac Cardiovasc Surg 1961;42:258-62.

7 Meng CC, Eckner FA, Lev M. Coronary artery distribution in tetralogy of Fallot. Arch Surg 1965;90:363.

8 Carvalho JS, Silva CMC, Rigby ML, et al. Angiographic diagnosis of anomalous coronary artery in tetralogy of Fallot. Br Heart f 1993;70:75-8.

9 O'Sullivan J, Bain H, Hunter S, et al. End-on aortogram: improved identification of important coronary artery improved identification of important coronary artery

10 McAlpine WA. The coronary arteries. In: McAlpine WA. Heart and coronary arteries. Berlin: Springer-Verlag, 1979: $140,201$.

11 Muriago M, Sheppard MN, Ho SY, et al. The location of the coronary arterial orifices in the normal heart. Clin Anat 1997;10:1-6.

2 Angelini P. Normal and anomalous coronary arteries: definitions and classification. Am Heart F 1989;117:418-28.

13 Shrivastava S, Edward JE. Coronary arterial origin in persistent truncus arteriosus. Circulation 1977;55:551-4.

14 Mustafa I, Gula G, Radley-Smith R, et al. Anomalous origin of the left coronary artery from the anterior aortic sinus: a potential cause of sudden death. F Thorac Cardiovasc Surg 1981;82:297-300.

15 Benson PA. Anomalous aortic origin of the coronary artery with sudden death: case report and review. Am Heart $\mathcal{f}$ 1970;79:254-7.

16 Cohen LS, Shaw LD. Fatal myocardial infarction in an 11 year old boy associated with unique coronary artery anomaly. Am f Cardiol 1967;19:420-3. 
17 Cheitlin MD, De Castro CM, McAllister HA. Sudden death as a complication of left coronary origin from the anterior
sinus of Valsalva. Circulation 1974;50:780-6.

18 Sim EKW, van Son JAM, Julsrud PR, et al. Aortic intramural course of the left coronary artery in dextro-transposition of the great arteries. Ann Thorac Surg 1994;57:458-60.

19 Asou T, Karl TR, Pawade A, et al. Arterial switch: translocation of the intramural coronary artery. Ann Thorac Surg 1994;57:461-5.

20 Pasquini L, Parness IA, Colan SD, et al. Diagnosis of intramural coronary artery in transposition of the great arteries using two-dimensional echocardiography. Circulation 1993 88:1136-41.

21 Baroldi G, Scomazzoni G. Coronary circulation in the normal and pathologic heart. Washington, DC: Department of the Army, United States Government Printing Office, 1967:590.

22 Helfant RH, Banka VS. A clinical and angiographic approach to coronary heart disease. Philadelphia: FA Davis Co, 1978:23.

23 Gensini GG. Coronary arteriography. Mt. Kisco, New York: Futura Publishing Co, 1975:168.

24 Humes RA, Driscoll DJ, Danielson GK, et al. Tetralogy of Fallot with anomalous origin of anterior interventricular coronary artery. Surgical options. $\mathcal{F}$ Thorac Cardiovasc Surg 1987;94:784-7.
25 Stellin G, Milanesi O, Rubino M, et al. Repair of tetralogy of Fallot in the first six months of life: transatrial versus transventricular approach. Ann Thorac Surg 1995;60:8588-91.

26 Jureidini SB, Scott Appleton R, et al. Detection of coronary artery abnormalities in tetralogy of Fallot by twodimensional echocardiography. $\mathcal{F}$ Am Coll Cardiol 1989;14: 960-7.

27 Berry JM, Einzig S, Krabill KA, et al. Evaluation of coronary artery anatomy in patients with tetralogy of Fallot by two56.

28 Santoro G, Marino B, Di Carlo D, et al. Echocardiographically guided repair of tetralogy of Fallot. Am $\mathcal{F}$ Cardiol 1994;73:808-11.

29 Stein PD, Sabbah HN. Orifice-view roentgenography for evaluation of the aortic valve. Am F Roentgenol Radium Ther Nuclear Med 1975;125:847-53.

30 Mandell VS, Lock JE, Mayer JE, et al. The "laid-back" aortogram: an improved angiographic view for demonstration of coronary arteries in transposition of the great arteries. Am f Cardiol 1990;65:1379-83.

31 Kaushai SK, Iyer KS, Sharma R, et al. Surgical experience with total correction of tetralogy of Fallot in infancy. Int $\mathcal{F}$ Cardiol 1996;56:35-40. 